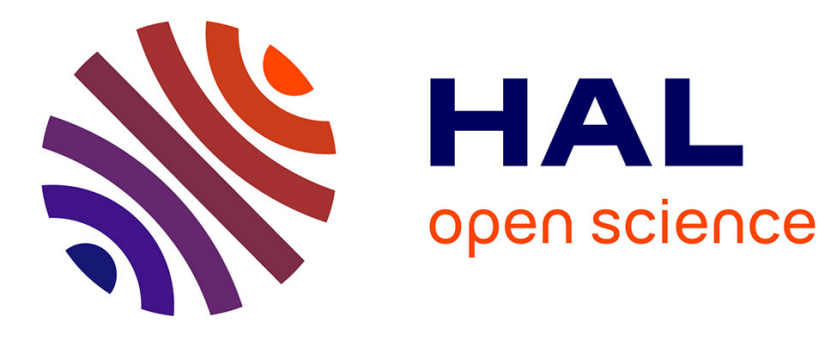

\title{
La réalité virtuelle et son intégration dans un projet Philippe Fleury
}

\section{To cite this version:}

Philippe Fleury. La réalité virtuelle et son intégration dans un projet. Les Nouvelles de l'archéologie, 2010, La médiation en archéologie, 122, pp.29-33. 10.4000/nda.1257 . hal-01609463

\section{HAL Id: hal-01609463 https://hal.science/hal-01609463}

Submitted on 3 Oct 2017

HAL is a multi-disciplinary open access archive for the deposit and dissemination of scientific research documents, whether they are published or not. The documents may come from teaching and research institutions in France or abroad, or from public or private research centers.
L'archive ouverte pluridisciplinaire HAL, est destinée au dépôt et à la diffusion de documents scientifiques de niveau recherche, publiés ou non, émanant des établissements d'enseignement et de recherche français ou étrangers, des laboratoires publics ou privés. 


\section{La réalité virtuelle et son intégration dans un projet}

Philippe Fleury*

Voir, depuis les années 1970, les colloques internationaux annuels du Computer Applications and Quantitative Methods in Archaeology (CAA - sommaires en ligne depuis 2000) et, depuis 2003, le colloque bisannuel "Virtual Retrospect" organisé à Bordeaux par R. Vergnieux et son équipe.

* Université de Caen, directeur du Centre interdisciplinaire de réalité virtuelle (CIREVE), philippe.fleury@unicaen.fr.
$\mathrm{L}$ a réalité virtuelle entre dans le large cadre des applications de l'informatique à l'archéologie ${ }^{1}$. Les fouilles menées depuis plus de deux siècles ont conduit à un accroissement considérable des collections, rendant leur gestion et leur exploitation de plus en plus difficiles : l'informatique documentaire et les systèmes de gestion des bases de données y apportent une aide matérielle non négligeable. Sur le terrain, la découverte de l'objet rare a cessé d'être un but et la tendance actuelle est à l'analyse. À partir des acquis et des pièces déjà rassemblées, il s'agit de réévaluer les hypothèses avancées, d'aboutir à leur vérification ou d'en formuler de nouvelles : l'informatique (associée à la notion de modèle) apporte les capacités de calcul et la rigueur de la démarche. Quand il faut chercher les moyens les plus efficaces pour mettre en valeur, exploiter scientifiquement ou conserver le patrimoine - les monuments en particulier connaissent aujourd'hui de plus en plus d'agressions dues à la pollution, à l'urbanisme ou au tourisme de masse -, l'informatique apporte des solutions pour acquérir des images, les traiter, les présenter et les diffuser. L'exemple qui va être présenté ici, la restitution virtuelle de la Rome antique (Madeleine \&t Fleury 2007), participe de toutes ces démarches mais nous allons nous concentrer sur l'intégration de la réalité virtuelle dans le projet. L'université de Caen Basse-Normandie possède en effet un objet patrimonial exceptionnel, un plan-relief de la Rome du IV siècle après J.-C. réalisé par l'architecte Paul Bigot au début du $\mathrm{Xx}^{\mathrm{e}}$ siècle. Autour de cet objet, communément appelé le "Plan de Rome²", a été monté en 1994, dans le cadre de la Maison de la recherche en sciences humaines, un projet de valorisation incluant à la fois une mise en scène de la maquette et la réalisation de son double virtuel. De nouvelles étapes ont été franchies, en 2006, avec la création d'un Centre de réalité virtuelle (le CIREVE, http://www.unicaen.fr/cireve), puis, en 2008, avec celle d'une Équipe de recherche technologique éducation "Sources anciennes, multimédias et publics pluriels» (L'ERSAM, http://www.unicaen.fr/ersam). Ces dernières étapes ont permis d'intégrer la réalité virtuelle proprement dite dans le travail de restitution. Nous commencerons par poser les cadres conceptuels de la démarche scientifique en distinguant clairement la "réalité virtuelle" de la "restitution virtuelle". Nous montrerons ensuite comment le travail de restitution est depuis longtemps et en lui-même (bien avant l'invention de l'informatique...) un moyen de médiation des connaissances sur l'architecture et la topographie de la Rome antique. Nous terminerons par la "plus-value" de la réalité virtuelle dans le travail de restitution.

\section{La réalité virtuelle : définition et enjeux}

L'avènement de ce qui est communément appelé «la réalité virtuelle» s'est opéré dans les années 1980, parallèlement à l'essor de l'informatique graphique. Deux notions fondamentales lui sont attachées : l'immersion et l'interaction. L'immersion consiste à passer de l'autre côté du "miroir", c'est-à-dire à entrer dans l'image. Cette opération est physique lorsqu'une interface (un visiocasque par exemple) est utilisée mais elle peut être simplement mentale lorsque le spectateur observe une image tridimensionnelle sur l'écran et s'y projette mentalement. L'interaction (ou interactivité) consiste à interagir en temps réel avec l'image pour la manipuler ou la transformer, une des fonctions de base de toute application informatique (Fuchs 2006 et 2009; Cadoz 1994 ; Burdea \&t Coiffet 1993 ; Quéau 1993 ; Pimentel-Texeira 1994).

La réalité virtuelle repose sur l'image et, là aussi, quelques précisions sont nécessaires : qu'entend-on par "image de synthèse», "image numérique» ou "image 
virtuelle»? L'image de synthèse est entièrement produite sur ordinateur, sans transfert du réel. Par définition, elle est numérique, c'est-à-dire que ses composants sont codés pour être "lus" par un ordinateur. Mais, l'image numérique peut aussi provenir de la réalité qui a été numérisée, soit par scannérisation soit par saisie directe avec un appareil numérique. Si elle ne subit pas de modification, elle reste une "image réelle». En revanche, si elle est modifiée par ajout, suppression ou transformation d'éléments, elle devient une image virtuelle (l'image de synthèse est aussi par définition virtuelle).

Dans le domaine de la représentation archéologique, il $\mathrm{y}$ a fondamentalement trois modes d'utilisation de l'image virtuelle : l'image fixe, l'animation de synthèse et la visite interactive qui seule utilise la technique de la réalité virtuelle. L'image fixe est couramment utilisée dans les publications et sur l'Internet. L'animation de synthèse est une "visite guidée» : c'est l'enregistrement et la restitution sur un support numérique ou analogique d'une succession d'images virtuelles précalculées. Le temps de calcul nécessaire pour chaque image est théoriquement illimité mais, selon la norme utilisée il faut 24, 25 ou 30 images par seconde pour produire une animation fluide... Ces images peuvent être extrêmement réalistes et faire appel aux techniques les plus sophistiquées de l'informatique graphique. Une même séquence peut mêler des images de synthèse et des images réelles numérisées sans que le spectateur puisse faire la différence (le principe est maintenant couramment utilisé au cinéma). La visite interactive est la restitution instantanée des images virtuelles nécessaires à la simulation de l'environnement dans lequel évolue le (ou les) intervenant(s). Ces images ont besoin d'être traitées en temps réel. Pour garantir le sentiment d'immersion, le décalage entre l'action réelle et sa traduction virtuelle ne doit pas dépasser quelques dizaines de millisecondes. Dans ce laps de temps, l'ordinateur doit acquérir les données en provenance des capteurs (il peut s'agir du clavier ou d'une simple souris), les traiter, recalculer l'image correspondant à la nouvelle position et l'afficher, parfois la transmettre à distance. Les unités centrales et les systèmes graphiques sont exploités au maximum de leurs possibilités mais, dans l'état actuel des techniques, il faut se contenter d'images moins complexes et donc potentiellement moins réalistes que dans l'animation de synthèse.

L'interactivité pose enfin le problème du comportement des objets : un mur peut-il être traversé? L'intervenant peut-il s'élever au-dessus du sol sans support?... Dans le cas de l'animation de synthèse, le scénariste de la visite guidée fait un choix préalable : il trace le cheminement de la caméra virtuelle selon l'idée qu'il se fait du produit final. Mais, dans le cas de la visite interactive, le choix du trajet appartient à l'intervenant. Donc, si le concepteur de la base de données numérique qui servira de terrain à la visite veut vraiment simuler un monde réel, il doit donner aux objets un potentiel de réaction : un mur ne peut être traversé, une porte s'ouvre et offre une résistance en fonction de sa masse... Toutes ces données sont incluses dans la base et prises en compte par le logiciel d'interactivité.
L'application des méthodes de la réalité virtuelle à la restitution de la Rome antique réunit deux cultures, deux modes de pensée très différents, disons pour simplifier les cultures scientifique et humaniste. D'un côté, sont concernés les informaticiens et les spécialistes de l'image, de l'autre, ceux que l'on appelle communément les "antiquisants" : philologues, archéologues et historiens. Les uns et les autres ne peuvent travailler indépendamment sur ce sujet : les analyses historique et infographique sont intimement liées. Mais, à ce noyau, viennent aussi s'adjoindre d'autres disciplines comme la psychologie (particulièrement active dans le domaine de l'étude et de l'expérimentation du comportement en milieu virtuel) et, naturellement, les sciences de la communication.

\section{La restitution comme moyen de médiation}

En histoire et en archéologie, la restitution a toujours été utilisée comme adjuvant ou comme moyen de la transmission des connaissances sur des objets disparus, endommagés ou difficilement accessibles. Il s'agit en somme, à partir de l'analyse des sources anciennes textuelles, archéologiques ou iconographiques, de reproduire sur des supports variés des outils, des machines, des constructions, des environnements reconstruits d'abord mentalement.

La Rome antique, outre l'intérêt intrinsèque qu'elle suscite dans l'imaginaire collectif du monde occidental, est un sujet d'étude particulièrement intéressant dans le domaine de la restitution puisqu'elle offre tous les cas possibles de la transmission d'un patrimoine : disparition complète de bâtiments, état de ruines, conservation presque intacte, réutilisation et remaniement du bâtiment pour un autre usage, permanence viaire dans certains quartiers, bouleversement complet dans d'autres, etc. À partir du $\mathrm{v}^{\mathrm{e}}$ siècle après J.-C. en effet, la Rome dite "antique" disparaît sous l'effet des destructions barbares du temps et, surtout, sous l'effet de l'autodestruction. Elle fait place à une Rome chrétienne pour laquelle les monuments antiques sont au mieux objets de récupération - le temple de Portunus, la Curie, le Panthéon par exemple sont transformés en églises -, au pire objets de pillages ou de désintérêt - le centre historique perd son caractère monumental, les marbres antiques sont réutilisés dans d'autres structures ou transformés en chaux.

Les formes prises par la restitution de la Rome antique ont été diverses. Il peut s'agir d'une simple description textuelle, support choisi par Flavio Biondo, le premier "restaurateur" de la Rome antique, pour la publication de Roma instaurata en 1446 (Raffarin-Dupuis 2005). Pour la première fois, le travail de restitution est fait de façon scientifique, en confrontant sources archéologiques et sources textuelles. Plus couramment, la restitution est graphique : dessin au trait, sciographie ou aquarelle, projection orthographique ou vue en perspective... De la fin du XvIII ${ }^{\mathrm{e}}$ siècle jusqu'au début du $\mathrm{Xx}^{\mathrm{e}}$ siècle, les architectes ayant obtenu le "Grand Prix de Rome" ont eu comme exercice obligé à la fin de leur séjour à la villa Médicis de faire le relevé d'un bâtiment antique, mais aussi d'en proposer une restitution, accompagnée d'un mémoire explicatif (Pinon-Amprimoz 1988). Ces travaux, envoyés à Paris, 
forment la collection des "Envois de Rome». Aujourd'hui, chacun connaît l'œuvre de Jean-Claude Golvin, architecte, parfait connaisseur de l'Antiquité et aussi merveilleux aquarelliste (voir par exemple Golvin 2008). Mais, il arrive également que la restitution soit physique : anastylose, c'est-à-dire un remontage des éléments

3.

Les ruines actuellement visibles

du temple de Vesta à Rome, par exemple, sont le résultat d'une anastylose partielle (parfois contestée) réalisée avec des fragments originaux, complétée par des restaurations en travertin. effondrés avec ou sans remplacement des parties manquantes ${ }^{3}$, ou reproduction à l'échelle ou en modèle réduit. $\mathrm{Au} \mathrm{Xx}^{\mathrm{e}}$ siècle, deux grandes maquettes de Rome à l'époque de Constantin ont été réalisées : la maquette de Paul Bigot, conservée à l'université de Caen et dont une copie est exposée aux musées royaux d'Art et d'Histoire de Bruxelles (Royo 2006), et la maquette d'Italo Gismondi exposée au musée de la Civilisation romaine de Rome (Liberati 2003 ; Pavia 2008). Ces reproductions sont une forme d'achèvement : la somme des connaissances littéraires, archéologiques et iconographiques accumulées sur la Rome antique depuis la Renaissance.

L'intérêt médiatique de ces différentes restitutions, qu'elles soient sous forme de plans, de gravures, d'aquarelles ou de maquettes, n'est plus à démontrer. Ce sont souvent des objets d'art, mais, dans le domaine scientifique, les archéologues ou les historiens les utilisent aussi pour appuyer ou illustrer leurs hypothèses. Les élèves ou le grand public sont souvent fascinés par la maquette dans laquelle ils voient la Rome de leurs études "recréée». D’un point de vue pédagogique, c'est aussi un excellent outil de médiation.

Nous allons maintenant essayer de voir la "plus-value" apportée par la restitution virtuelle et, à l'intérieur de celle-ci, par l'intégration de la réalité virtuelle proprement dite.

\section{Réalité virtuelle et restitution}

Le point de départ, le prétexte du travail de restitution virtuelle mené à Caen, est donc la maquette de la Rome du IV siècle après J.-C. réalisée au 1/400, entre 1900 et 1940, par Paul Bigot, un architecte normand. Il s'est appuyé sur des données archéologiques, littéraires et historiques, et a utilisé en particulier les travaux alors en plein renouveau sur la Forma Vrbis, plan gravé sur marbre à l'époque de Septime Sévère (fin $\mathrm{II}^{\mathrm{e}}$-début $\mathrm{III}^{\mathrm{e}}$ siècle après J.-C.) et dont un dixième seulement a été retrouvé au $\mathrm{XvI}^{\mathrm{e}}$ siècle. Paul Bigot fit une œuvre dont la valeur artistique, pédagogique et scientifique fut rapidement reconnue.

Après avoir travaillé quarante années sur sa maquette, participé à plusieurs expositions importantes et réalisé trois copies, Paul Bigot légua l'original à l'un de ses disciples, Henri Bernard, l'architecte chargé de la reconstruction de l'université de Caen après la deuxième guerre mondiale. Celui-ci en fit don à l'université et une salle spécialement aménagée l'abrita dans les sous-sols de la faculté de droit. En 1995, après une importante restauration, la maquette fut installée au cœur d'un nouveau bâtiment de l'université de Caen, la Maison de la recherche en sciences humaines.
Le contexte était alors favorable à la fois pour créer une dynamique de mise en valeur de l'objet et pour ouvrir de nouvelles perspectives de recherche.

Pour mener ce travail, fut créé un pôle pluridisciplinaire de recherche d'abord intitulé "Architecture, urbanisme et image virtuelle - La Rome antique» puis, avec une perspective plus large "VILLE - Architecture, urbanisme et image virtuelle». Ce pôle regroupait des enseignants-chercheurs, des ingénieurs et des étudiants de troisième cycle. Les disciplines représentées étaient, pour le noyau de base, les sciences de l'Antiquité, l'informatique et l'audiovisuel, mais les séminaires et la collaboration avec d'autres institutions étendirent la représentation à la géographie, l'urbanisme, la littérature, les beaux-arts, l'architecture... Trois institutions françaises collaborèrent étroitement avec le pôle : l'École d'architecture de Marseille (Groupe d'application des méthodes scientifiques à l'architecture et à l'urbanisme - GAMSAU), l'École navale de Brest (Réalité virtuelle et simulation) et l'École des beaux-arts de Caen (Infographie).

En 2006, l'équipe travaillant sur le Plan de Rome fut à l'origine de la création du CIREVE, service commun de l'université de Caen Basse-Normandie, dont les missions principales sont : la mutualisation des équipements de réalité virtuelle (cluster, projecteurs, écran, interfaces...), la mutualisation des compétences et l'organisation de formations, la promotion et le soutien de l'utilisation de la réalité virtuelle dans la recherche aussi bien pour les sciences humaines et sociales que pour les sciences exactes et expérimentales ${ }^{4}$.

En 2008, le dispositif fut complété par l'ERSAM dont l'objectif est de rendre accessible les sources anciennes à de larges publics en utilisant les technologies multimédias. À l'intérieur de cette équipe se poursuivent aujourd'hui le travail de restitution virtuelle de la Rome antique et l'intégration de la réalité virtuelle dans le projet.

La maquette virtuelle de la Rome antique réalisée à Caen est d'abord un modèle numérique construit à partir des sources textuelles, archéologiques et iconographiques disponibles. L'époque de l'empereur Constantin, période également choisie par Paul Bigot et Italo Gismondi, permet de représenter la ville de Rome à son apogée monumental et surtout dans son dernier état antique, celui que redécouvriront les hommes de la Renaissance et qui sera la source d'inspiration des architectes occidentaux. C'est ensuite un modèle dont l'apparence est la plus soignée possible, avec des éclairages et des textures réalistes ${ }^{5}$. C'est enfin un modèle auquel sont associées les sources qui ont permis de le constituer.

Les textures sont la représentation des matériaux qui "habillent» le modèle pierres, bois, mosaïques, enduits, etc

L'objectif de l'équipe caennaise est de contribuer à la connaissance scientifique d'une ville dont l'architecture et l'urbanisme, à la fois cadres et expressions de la civilisation, ont marqué l'Occident de façon indélébile. La maquette virtuelle offre un double intérêt. D'un point de vue scientifique, elle donne la possibilité d'une mise à jour permanente. Depuis 


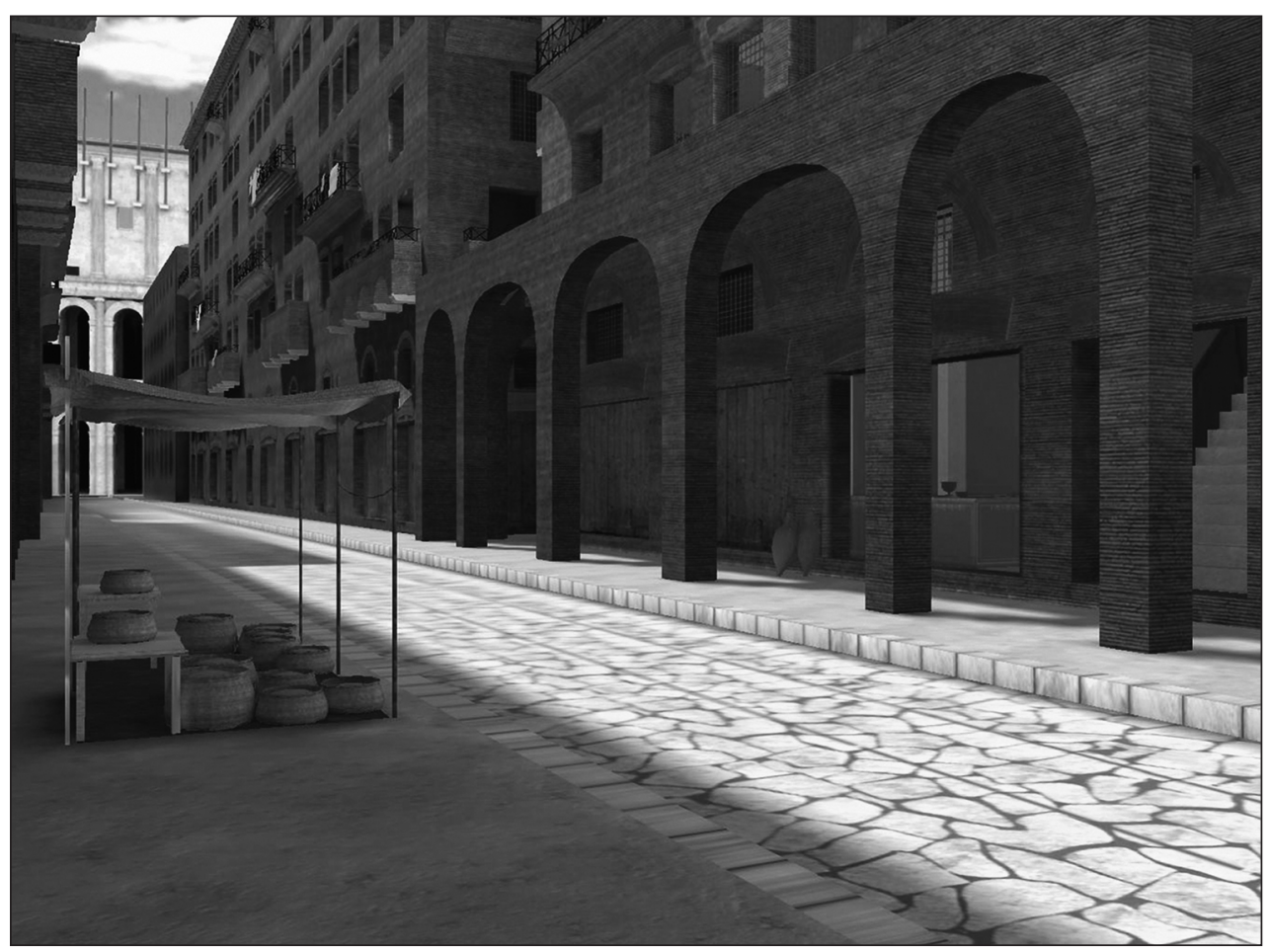

Fig. 1 - Une rue de Rome,

vue subjective.

les métadonnées au fichier de restitution lui-même. En pointant avec la souris sur les différentes parties de l'image virtuelle, l'utilisateur accède directement aux sources qui ont permis la restitution : outre la bibliographie moderne qui a été utilisée, il trouve les principales sources archéologiques, iconographiques et

le début du travail, en 1994, presque tous les monuments réalisés ici ont été modifiés à la suite de la prise en compte d'éléments nouveaux ou de la réinterprétation d'éléments anciens. En dehors de la mise à jour nécessaire, la maquette virtuelle est aussi un terrain d'expérience, de confrontation des hypothèses, de test des systèmes mécaniques associés à l'architecture (vélums des édifices de spectacle, rideau de scène, systèmes de levage, élévation d'eau, etc.) et un espace de visite. La réalité virtuelle prend tout son sens grâce à l'immersion et à l'interactivité. L'utilisateur n'est plus face à un modèle réduit, comme dans le cas de la maquette physique, il est à l'échelle de la ville. Il voit Rome en stéréoscopie ou en monoscopie selon le système utilisé, du point de vue de son regard (vue subjective - fig. 1) ou en suivant un avatar à quelques mètres (vue objective - fig. 2). Il peut se déplacer librement dans le modèle, accéder à l'intérieur des monuments publics et voir leur aménagement et leur décoration, accéder aussi à l'intérieur de quelques habitations privées de différents types (domus ou insulae). Pour être scientifique, une image de restitution doit informer l'utilisateur sur les différents degrés de fiabilité de la restitution selon les parties de l'objet. Mais, pour répondre à un spectre élargi d'utilisations (perspectives scientifique, pédagogique et esthétique), l'image de restitution doit être complète. Les solutions graphiques qui consistent à matérialiser les degrés de fiabilité en chargeant la représentation elle-même d'éléments critiques (couleurs différentes, grisés, pointillés, lignes seules pour les parties mal ou non informées...) ne sont en général ni suffisantes pour une exploitation scientifique, ni parlantes pour une utilisation pédagogique, ni même belles. Pour résoudre ce paradoxe, le modèle interactif de la Rome antique utilise la possibilité offerte par les technologies multimédias de lier textuelles qui ont servi de point d'appui et il peut ainsi juger de son degré de fiabilité. L'accès direct à la documentation à partir du modèle permet d'utiliser celui-ci comme une publication scientifique : le renvoi aux sources et aux différentes hypothèses joue alors le rôle des notes ou de l'apparat critique. La réalité virtuelle produit ainsi un véritable outil de représentation accessible à des publics pluriels : tandis que le spécialiste y voit une hypothèse de restitution justifiée, le non-spécialiste trouve tout simplement la visualisation d'une réalité qu'il peine à imaginer par la description textuelle, le plan ou même le dessin en deux dimensions.

Les deux maquettes caennaises, en plâtre et virtuelle, ne s'opposent pas mais se complètent, se répondent. Les "nouvelles technologies" (pour reprendre cette expression consacrée) offrent en fait quatre nouvelles possibilités : 1) la quasi-immatérialité du support ${ }^{6}$ pour des modifications relativement aisées et un stockage pratiquement infini des différentes versions ${ }^{7}$; 2) la réalité virtuelle grâce à laquelle on s'immerge à "échelle humaine" dans le modèle et on interagit avec lui; 3) le multimédia qui associe l'image (animée ou fixe), le texte (par exemple les sources anciennes sur lesquelles s'appuie la restitution) et le son; 4) l'accès à distance via l'In- 
Fig. 2 - Le portique du forum d'Auguste avec l'avatar, vue objective.

ternet. Mais, en aucun cas, la maquette virtuelle ne rend obsolète la maquette physique qui reste un objet d'art classé "monument historique" et un témoin des connaissances sur Rome de la première moitié $\mathrm{du} \mathrm{xx}^{\mathrm{e}}$ siècle. Le travail de restitution virtuelle est

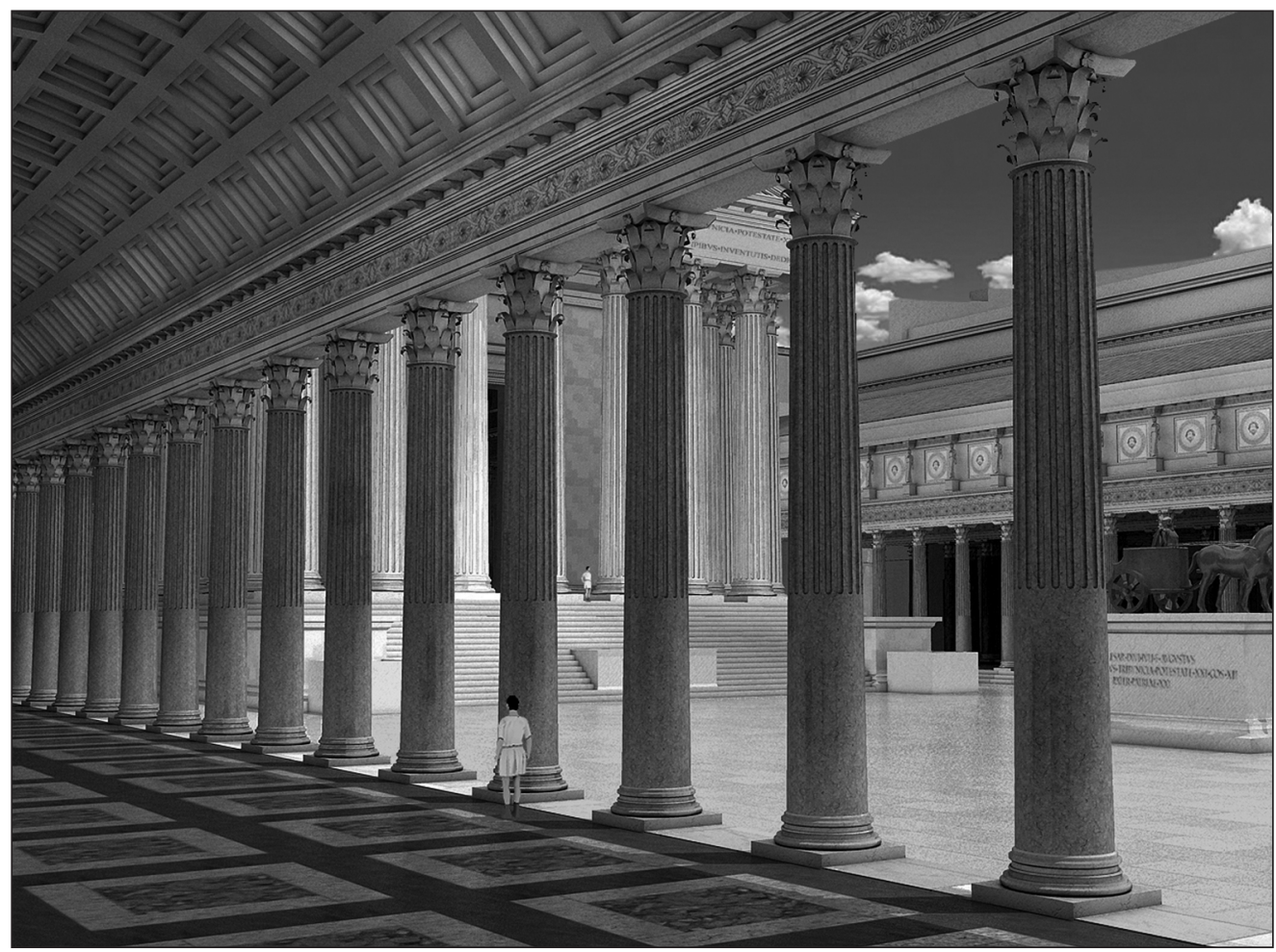
encore à compléter et à améliorer mais l'intégration de la réalité virtuelle offre déjà une nouvelle forme d'attrait pour les études classiques comme en témoigne le succès des Nocturnes du Plan de Rome (présentations mensuelles d'une partie de la maquette virtuelle en stéréoscopie), organisées à l'université de Caen Basse-Normandie et ouvertes au grand public. C'est aussi un objet de focalisation de la recherche internationale autour de quelques aspects des sciences de l'Antiquité : un travail d'une telle ampleur ne saurait être réalisé sans des échanges avec des chercheurs du monde entier. L'équipe caennaise collabore depuis l'origine avec celle de R. Vergnieux de l'Institut "Ausonius" (université de Bordeaux-III) et une convention signée en 2008 avec l'équipe "Rome Reborn» dirigée par B. Frischer (université de Virginie, UsA) associe dorénavant les efforts des deux équipes engagées dans une restitution globale de la Rome de Constantin. C'est enfin un moyen de rendre lisible et de valoriser la recherche sur l'Antiquité à travers les nouveaux médias.

\section{Références bibliographiques}

BurdeA, G. \& Ph. Colffet. 1993. La réalité virtuelle. Paris, Hermès. CADOZ, C. 1994. Les réalités virtuelles. Paris, Flammarion (Dominos). Computer Applications and Methods in Archaeology. 1970 et suivants.

Fuchs, Ph. (dir.). 2006 et 2009. Le traité de la réalité virtuelle. $3^{e}$ édition, Paris, Presses de l'École des mines, vol. 1-4 : 2006, vol. 5 : 2009.

GoLvIN, J.-Cl. 2008. Rome antique retrouvée. Paris, Errance.

LIBERATI, A.-M. 2003. "La rappresentazione di Roma antica nel plastico di Gismondi del Museo della civilità romana a Roma», in : "Rome an 2000 - Ville, maquette et modèle virtuel», Les Cahiers de la MRSH, 33 : 243-252.

MAdeLEINE, S. \& Ph. FLEURY. 2007. «Réalité virtuelle et restitution de la Rome antique au Ive siècle après J.-C.n, Histoire urbaine, 18 : 161-169.

PaviA, C. 2008. "La riscostruzione dell'antica Roma. Breve storia del platiscismo romano", Forma Vrbis, XIII, 5 : 30-39.

Pimentel, K. Et K. TeXeira. 1994. La réalité virtuelle. De l'autre côté du miroir. Paris, Addison-Wesley (édition originale : Virtual Reality, Beyond the New Looking Glass, 1993).

Pinon, P. \&t F.-X. Amprimoz. 1988. Les Envois de Rome (1778-1968). Architecture et archéologie. Rome, École française de Rome (Collection de l'École française de Rome; 110).

QuÉAU, Ph. 1993. Le virtuel. Vertus et vertiges, Seyssel, Champ Vallon - INA.

RafFarin-Dupuis, A. (éd., trad., prés. et notes). 2005. Flavio Biondo, Roma instaurata, Livre I. Paris, Les Belles Lettres.

Royo, M. 2006. Rome et l'architecte : conception et esthétique du planrelief de Paul Bigot. Caen, Presses universitaires de Caen.

VergnieuX, R. (dir.), Virtual Retrospect 2003 et suivants, université de Bordeaux. 